\title{
Consideraciones clínicas en la obtención de injertos óseos intraorales. Técnica quirúrgica y evaluación de las complicaciones
}

\author{
Considerations for intraoral osseous graft remove. Technique and \\ complications management
}

\author{
ROCHA F* \\ DE OLIVEIRA GR ** \\ OLATE S*** \\ DE ALERGARIA-BARBOSA JR****
}

\begin{abstract}
Rocha F, de Oliveira GR, Olate S, de Alergaria-Barbosa JR. Consideraciones clínicas en la obtención de injertos óseos intraorales. Técnica quirúrgica y evaluación de las complicaciones. Av Periodon Implantol. 2010; 22, 2: 71-76
\end{abstract}

\begin{abstract}
RESUIMEN
Las técnicas de remoción de injertos intraorales están bien descritas en la literatura internacional; sin embargo, siempre es importante recordar las condiciones que pueden influir en la presencia de complicaciones transquirúrgicas y postquirúrgicas. Por otra parte, las técnicas de reconstrucción ósea son realizadas por muchos cirujanos dentistas, con diferentes grados de formación, lo que puede permitir el desconocimiento de algunos puntos importantes cuando se realice el procedimiento quirúrgico.

Los autores realizan una revisión de la literatura con énfasis en las condiciones de la técnica quirúrgica, tipos de complicaciones y su manejo.

Finalmente, podemos señalar que después de protocolizar las técnicas quirúrgicas, el siguiente paso es el manejo de las complicaciones, que pueden disminuir con una correcta conducta quirúrgica.
\end{abstract}

PALABRAS CLAVE: Injertos óseos, técnica quirúrgica, complicaciones quirúrgicas.

\section{SUMMARY}

A technique for intraoral graft remove are well described in the international literature, but always is important to remember the conditions that can influenced for complications in surgical and post surgical time. Considering this, the osseous graft technique are be executed for many dental practitioner, with different type of formation, that can lead to ignorance of some the moment of to realize the surgical procedure.

The author's realize a literature review with emphasis in the conditions of the surgical technique, type of complications and your management.

Finally, can be say that after of construed a surgical protocol, the protocol for complications management can be the next way. The complications could be diminished with a properly surgical procedure.

KEY WORDS: Osseous graft, surgical technique, complications.

Fecha de recepción: 14 de abril de 2009.

Fecha de aceptación: 25 de abril de 2009.

* CD. Práctica Privada.

** CD, MS, PhD. Profesor Adjunto. División de Cirugía y Traumatología Buco-Maxilo-Facial. Universidad Estadual do Oeste do Paraná. Brasil.

*** CD, MS. Profesor. Facultad de Medicina. Universidad de La Frontera. Chile. Alumno de Doctorado, Cirugía y Traumatología Buco-Máxilo-Facial. Facultad de Odontología de Piracicaba. Universidad Estadual de Campinas. Brasil.

**** CD, MS, PhD. Profesor Titular. División de Cirugía y Traumatología Buco-Maxilo-Facial. Facultad de Odontología de Piracicaba. Universidad Estadual de Campinas. Brasil. 


\section{INTRODUCCIÓN}

El injerto óseo más antiguo del que se tiene datos viene del año 1682, cuando Van Meeken trasplantó hueso del cráneo de un perro para un defecto craniano en un hombre (1-3). Adell (4) y Braine (5) fueron los primeros en estudiar el uso de injertos autógenos asociados a implantes de titanio en maxilares atróficos; Brånemark, sin embargo, describió la técnica ya en el año 1975.

Después de la pérdida dentaria, se puede observar la reabsorción del reborde alveolar, especialmente en las regiones vestibulares y en relación a la altura del mismo (6); esta reabsorción es siempre de mayor grado en la región maxilar y también es de mayor grado en regiones posteriores, donde se mantiene el ancho óseo con más frecuencia (7).

Los sitios donantes de la cavidad oral más utilizados en reconstrucciones óseas son del ramo mandibular, sínfisis mandibular y tuberosidad maxilar, siendo las complicaciones más frecuentes tratadas con medidas locales y en los casos más complejos, con la ejecución de algún procedimiento quirúrgico adicional. El hueso autógeno es el único material capaz de aportar células vivas totalmente inmunocompatibles $(8,9)$, proporcionando formación ósea por la capacidad de promover osteoinducción, osteoconducción y osteogénesis.

La región maxilofacial, como área donante, presenta menor reabsorción y mejor vascularización (10-14). La mayor compatibilidad entre áreas donantes y áreas receptoras junto al mantenimiento del volumen óseo son favorecidas por factores como el mismo origen embrionario (ectomesénquima), mismo tipo de formación ósea (intramembranosa en maxilar y cuerpo mandibular) y un espesor cortical favorable para disminuir el nivel de reabsorción. Además, el hueso cortical presenta mayor concentración de proteína ósea morfogenética que favorece la osteoinducción. Actualmente se ha establecido que los injertos óseos sin estimulo mecánico adecuado sufren reabsorciones en torno al $92 \%$ (15) y que el hueso de origen intramembranoso $(13,16,17)$ tendría mayor tendencia a mantener el volumen con una revascularización más rápida cuando es comparada con injertos de origen endocondral.

La mayor parte de las complicaciones se relaciona con el sitio donante y generalmente son transitorias. Equimosis, hematoma, edema, ptosis labial, disturbios neurosensoriales, necrosis tisular, dehiscencia de suturas, exposición del injerto, entre otras, son complicaciones frecuentemente citadas en la literatura (18-21).

De acuerdo con Misch (22), las dimensiones mínimas del reborde alveolar que aceptaría la instalación de implantes serían de $5 \mathrm{~mm}$ de espesor y $10 \mathrm{~mm}$ de altura. Cuando esas medidas son menores, es necesario realizar algún procedimiento reconstructivo, donde los injertos obtenidos de maxilar presentan un índice de reabsorción cercano al 10\%, mientras que los de origen mandibular, $5 \%$ (23). La elección del sitio donante dependerá, finalmente, del volumen óseo necesario para el defecto a tratar (1).

El objetivo de este trabajo es evaluar las técnicas quirúrgicas más utilizadas en la recolección de injertos y conocer sus complicaciones más frecuentes.

\section{AREAS DONANTES INTRAORALES}

\section{Sínfisis}

El análisis con radiografía panorámica permite una evaluación general con las dimensiones laterales máximas probables, mientras que la radiografía lateral permite evaluar el espesor del hueso cortical disponible. Las radiografías periapicales permiten realizar una evaluación de la distancia de la osteotomía sobre los ápices radiculares (7).

La eliminación de hueso cortical y esponjoso, permite obtener una rápida revascularización y mantenimiento del volumen del hueso injertado (22-29). Osaki y Buchman (30) señalan que el éxito del procedimiento esta en relación con las consideraciones hacia los aspectos geométricos característicos de hueso cortical y esponjoso injertado. Park y cols (31) realizaron un estudio topográfico en 35 mandíbulas constatando que el máximo hueso cortical presente en un bloque rectangular de región de sínfisis pude obtenerse con dimensiones de 1-1,5 cm de altura, $4 \mathrm{~cm}$ de ancho, respetando los márgenes de $5 \mathrm{~mm}$ bajo las raíces y $5 \mathrm{~mm}$ sobre el límite basal de la mandíbula. También se observó que la cortical se torna más espesa desde superior hacia inferior, variando de $1,5 \mathrm{~mm}$ hasta un poco mas de $2 \mathrm{~mm}$.

El injerto extraído de sínfisis está indicado para pérdidas óseas pequeñas y medias, equivalente a 4 dientes en ancho o dos dientes en alto y ancho (7). En forma particular, puede emplearse en el seno maxilar o dentro de las cavidades resultantes de las enucleacio- 
nes quísticas. También puede emplearse para injertos en fisuras alveolares, reconstrucciones de suelo de órbita y como complemento en osteotomías tipo Le Fort I $(1,29)$. La eliminación de este injerto está indicada después de la erupción de los caninos, cuando es mayor la cantidad ósea que se puede obtener.

Mazzonetto y cols (21) utilizaron anestesia local con bloqueo del nervio alveolar inferior de forma bilateral. La incisión se realiza como mínimo $5 \mathrm{~mm}$ hacia inferior y anterior de la línea mucogingival. Es importante realizar un desbridamiento de tejidos hasta visualizar los nervios mentonianos, identificando los límites máximos de la osteotomía que serían $5 \mathrm{~mm}$ hacia anterior del foramen mentoniano.

Las principales complicaciones en este procedimiento son la equimosis en cuello, perforación de la cortical lingual, necrosis dentaria, ptosis de la musculatura labial, hematomas y posibles alteraciones en el perfil facial $(32,33)$. Las parestesias más frecuentes se asocian al nervio incisivo o a ramos terminales del nervio mentoniano, ocasionadas generalmente por el movimiento de eliminación del injerto (34). En un estudio con 27 pacientes con eliminación del injerto de mentón, 33\% presentó alteraciones neurosensoriales y después de 12 meses, sólo el 7,4\% mantenía esa queja, aunque con disminución progresiva de la parestesia (19).

\section{Rama mandibular}

Esta área se caracteriza por presentar una gran cantidad de hueso cortical y escaso hueso medular. Se realiza una osteotomía rectangular con $4 \mathrm{~mm}$ de espesura aproximadamente (25). Es útil en espacios edéntulos de 1 a 3 dientes, pudiendo utilizarse como bloque 0 como injerto particulado; es muy importante establecer la relación entre el bloque que se extrae y la posición del nervio alveolar inferior.

En comparación a la sínfisis, este área presenta menores índices de complicaciones postoperatorias como parestesias, dehiscencias de la herida y equimosis (10, 35-36), constituyéndose como un área muy utilizada en reconstrucción ósea (32).

Kubara y cols (1) utilizaron anestesia infiltrativa y bloqueo al nervio alveolar inferior, con una incisión que comienza en la base del ramo, caminando por la línea oblicua y con una extensión variable. Con fresas o sierras pueden realizarse las osteotomías que son ejecu- tadas en la región anterior vertical y superior e inferior horizontal; posteriormente se utilizan cinceles para la remoción del injerto. Las osteotomías se realizan hasta encontrar puntos muy sangrantes, señal de que está presente el hueso esponjoso (7)

\section{Tuberosidad maxilar}

Esta región presenta cantidades suficientes de hueso esponjoso, con una cortical muy fina; ocasionalmente puede presentar lugares con células que aumentarían el potencial osteogénico (25).

A través de una incisión lineal con o sin incisiones de descarga se extrae el tejido óseo con pinzas gubias, finalizándose con regularización de la estructura ósea y la aplicación de sutura simple; de esta forma, es la región donante de mas fácil acceso; el hueso recolectado se utiliza en forma particular estando indicado para cavidades óseas pequeñas y defectos pequeños $(1,25)$; sin embargo, por ser básicamente hueso esponjoso, presenta una tasa de reabsorción aumentada (10\% o más) (23).

\section{ALTERACIONES NEUROSENSORIALES}

Cada fibra de un nervio periférico se rodea por una lámina basal, fibras colágenas y capilares, formando un conjunto de tejido denominado endoneuro. El conjunto de fibras nerviosas se agrupan alrededor de una camada de tejido conjuntivo denominada perineuro, lo que ayuda a soportar el epineuro, que es la región más externa de un nervio, formado por tejido conectivo, vasos linfáticos y vasos sanguíneos, siendo responsable de soportar las tensiones y compresiones del medio; finalmente el mesoneuro es quien aporta la irrigación sanguínea; cualquier lesión en alguno de estos tejidos desencadena alteraciones nerviosas transitorias o permanentes (37).

Por ejemplo, con un hematoma y hemorragia de los vasos del epineuro llevan es necesario realizar compresión de fibras nerviosas causando neurotoxicidad localizada; la liberación de sangre y sus productos durante la formación del hematoma llevan a fibrosis y posterior compresión, disminuyendo la reparación natural del nervio (37).

La parestesia, hiperestesia e hipoestesia ocurren con la desmineralización parcial del nervio durante el proceso de cicatrización, lo que impide la conducción de 
parte de los impulsos nerviosos. Durante la regeneración, nuevas vainas de mielina pueden formarse aumentando el diámetro del axón. En estas condiciones, las áreas lesionadas comienzan a estimular la generación de dolor, denominándose parestesia o disestesia.

Se mantienen algunas alteraciones por la aprehensión del nervio por tejido cicatricial (38). Si se provoca alguna sensación por la estimulación mecánica en la región del nervio, es señal de buen pronóstico (39). Se espera la recuperación para después de días o meses, con lo que es posible obtener mejoras limitadas del cuadro (39).

\section{Tratamiento}

El láser de baja intensidad emite campos electromagnéticos que van desde el infrarrojo al ultravioleta. Cuando absorbe por el tejido, actúa excitando electrones y moléculas, promoviendo la estimulación e inhibición de reacciones químicas, estimulando funciones naturales de los tejidos (40). Las lesiones de grupos de fibras nerviosas externas con signos de parestesia pueden beneficiarse con este tratamiento. El éxito del tratamiento se relaciona con el tiempo desde que se inicio el tratamiento y el tiempo desde que inicio la patología (41).

El efecto del láser de baja intensidad se ha observado con una aceleración de la reparación tisular, disminución del dolor, restablecimiento de la función neural, estimulación para la liberación de endorfina, aumento de producción de mielina, promoción para la recuperación del axón, aumento de la microcirculación local y de la velocidad de cicatrización y aumento de la actividad metabólica neuronal $(38,40,41)$. El de tipo infrarrojo es el más indicado por presentar mayor penetrabilidad en los tejidos. Aumenta la permeabilidad de $\mathrm{Ca}^{2+}$ en la membrana celular, contribuyendo en la duplicación del ADN y en la replicación del ARN. (40).

La microcirugía está indicada en pacientes que no presentan mejora después de tres meses de aplicación de láser; en el caso del nervio alveolar inferior, la reparación quirúrgica debe realizarse antes de que ocurra la degeneración de la porción distal del mismo. Considerando que esta es lenta, la reparación quirúrgica es posible que se pueda realizar hasta seis meses después de ocurrida la lesión (42).

Cuando la alteración de la sensibilidad es transitoria y es debida principalmente a la reacción inflamatoria, está indicado el tratamiento con corticoesteroides y AINES (42). Sin embargo, no existe ningún protocolo farmacológico suficiente para el tratamiento de complicaciones nerviosas y hasta el momento no ha sido determinada evidencias de que el complejo B, asociado o no a corticoesteroides, tenga eficacia en el tratamiento de las parestesias (45).

\section{PREVENCIÓN DE LAS COIMPLICACIONES POSTOPERATORIAS}

La eliminación de hueso desde región de la tuberosidad maxilar podría permitir el desarrollo de algún tipo de comunicación bucosinusal. En esta condición clíni$\mathrm{ca}$, el cierre quirúrgico junto a la administración de antibióticos y descongestionantes es el tratamiento de elección $(1,43)$.

En la prevención de hematomas es importante evitar el mantenimiento de espacio ente capas de tejido blando, realizando una adecuada sutura por planos; en este sentido, la eliminación de injertos bicorticales en sínfisis está contraindicada (21). La exposición de injertos es otra complicación, que puede ser solucionada con nueva sutura o con la eliminación del injerto cuando esté indicado. Después de la eliminación de injertos de sínfisis, la sutura debería comenzar por la línea media, disminuyendo las tensiones del colgajo, previniendo la dehiscencia de la herida (33). Es utilizada sutura y compresión para impedir la ptosi labial e irregularidades en el surco mentolabial, aunque esta condición es aun controvertida (44).

\section{CONCLUSIÓN}

Están bien descritas las técnicas de recolección e instalación de injertos mientras que el manejo de complicaciones no está definido. Construir protocolos para tratar complicaciones es una necesidad y es el objetivo de nuevas investigaciones en desarrollo.

\section{BIBLIOGRAFÍA}

1. Kuabara MR, Vasconcelos LW, Carvalho PSP. Técnicas cirúrgicas para obtenção de enxerto ósseo autógeno. Rev Fac Odontol Lins/Unimep 2000;12:44-51.

2. Chase S, Herndon C. The fate of autogenous andhomogenous bone grafts: a historical review.J Bone Joint Surg 1955;37:809-41. 
3. Prolo DJ, Rodrigo JJ. Contemporary bone graft physiology and surgery. Clin Orthop 1985;200:322-42.

4. Adell R. Regeneration of the periodontium: an experimental study in dogs. Scand J Plast Reconst Surg 1974; 11(Suppl):1-177.

5. Breine U, Branemark P-I. Reconstruction of alveolar jaw bone. An experimental and clinical study of immediate and preformed autologous bone grafts in combination with osseointegrated implants. Scand. J Plast Reconstr Surg 1980;14:23-48.

6. Pietrokovski J, Massler M. Alveolar Ridge Resorption Following Tooth Extraction. J Prosthet Dent 1967;17:217.

7. Mish CE. Implantes Dentários Contemporâneos. Santos ed, Brasil, 2000, pp 451-67, 497-508.

8. Mazzonetto R, Passeri LA, Moreira RWF, ScheideggerSilva L. Avaliação da eficácia de enxertos de sínfise mandibular na reconstrução de defeitos ósseos na região anterior de maxila. Rev Bras Implant 2000; 1:19-22.

9. Olate S, Oliveira GR, Jaimes M. Cicatrización ósea en procedimientos de reconstrucción y colocación de implantes. Int J Morphol 2007;25:649-57.

10. Misch CM. Comparison of intraoral donor sites for ridge augmentation prior to implant placement. Int J Oral Maxillofac Implants 1997;12:767-76.

11. Misch CM, Misch CE, Resnik R, IsmailYH. Reconstruction of maxillary alveolar defects with mandibular symphysis grafts for dental implants: a preliminary procedure report. Int J Oral Maxillofacial Implants 1992; 7:360-6.

12. Zins JE, Whitaker LA. Membranous vs endochondral bone autografts: implications for craniofacial reconstruction. Plast Reconstruct Surg 1983;72:778-85.

13. Simth JD, Abramson M: Membranous vs. endochondral bone autografts. Arch Otolaryngol 1974;99:203-5.

14. Rabie ABM, Dan Z, Samman N: Ultrastructural identification of cells involves in the healing of intramembranous and endochondral bones. Int J Oral Maxillofac Surg 1996; 25:383-8.

15. Fazili M, Von Overvest-Eerdmans GR, Vernooy AM, Visser WJ, Von Waas MA. Follow-up investigation of reconstruction of the alveolar process in the atrophic mandible. Int J Oral Surg 1978;7:400-4.

16. Sindet-Pedersen and Enemark H. Mandibular Bone Grafts for Reconstruction of Alveolar Clefts. J Oral Maxillofac Surg 1988;46:533-7.

17. Kusiak JF, Zins JE, Whitaker LA. The early revascularization of membranous bone. Plast Reconstr Surg 1985;76:510.

18. Cordaro L, Sarzi Amadè D, Cordaro M. Clinical results of alveolar ridge augumentation with mandibular block bone grafts in partially edentulous patients prior to implant placement. Clin Oral Impl Res 2002;3:103-11.

19. Josh A. An investigation of post-operative morbidity following chin graft surgery. Br Dent J 2004;196:215-8.

20. Clavero J, Lundgren S. Ramus or chin grafts for maxillary sinus inlay and local onlay augumentation: comparison of donor site morbidity and complications. Clin Implant Dent Relat Res 2003;5:154-60.

21. Booij A, Raghoebar GM, Jansma J, Kalk WWI, Vissink A. Morbidity of chin bone transplants used for reconstructing alveolar defects in cleft patients. Cleft Palate Craniofac J 2005;42:533-8.

22. Misch CM, Misch CE, Resnik RR, IsmailYH. Reconstruction of maxillary alveolar defects with mandibular symphysis grafts for dental implants: a preliminary procedural report. Int J Oral Maxillofac Implants 1992;7:360-6.

23. Raghoebar G, Batenburg R, Vissink A, Reintsema H. Augmentation of localized defects of the anterior maxillary ridge with autogenous bone before insertion of implants. J Oral Maxillofac Surg 1996;54:1180-5.

24. Ozaki W, Buchman SR. Volume maintenance of onlay bone grafts in the craniofacial skeleton: micro architecture versus embryologic origin. Plast Reconstr Surg 1998;102:291-9.

25. Mathias RMV, Bassanta AD, Ramalho SA, Saba-Chujfi E, Simone JL. Enxertos autógenos com sítios doadores na cavidade oral. RGO 2003;51:249-56.

26. Yajima A, Otonari-Yamamoto M, Sano T, Hayakawa Y, Otonari T, Tanabe K, Wakoh M, Mizuta S, Yonezu H, NakagaWa K and Yajima Y. Cone-beam CT(CB Throne) Applied to Dentomaxillofacial Region. Bull Tokyo Dent Coll 2006;47:133-41. 
27. Scarfe WC, Farman AG, Sukovic P. Clinical Applications of Cone-Beam computed Tomography in Dental Practice. J Calif Dent Assoc 2006;72:75-80.

28. Weeraya T, Okouchi K, Fujiwara Y,Yamashiro M, Marouka Y, Ohbayashi N, Kurabayashi T. A comparative study of cone-beam computed tomography and conventional panoramic radiography in assessing the topographic relationship between the mandibular canal and impacted third molars. Oral Surg Oral Med Oral Pathol Radiol Endodon 2007;103:253-9.

29. Freitas RR, Ferreira da Silva AA, Borba M. A mandíbula como área doadora de enxertos em cirurgia bucomaxilo-facial. Rev APCD 2000;54:221-5.

30. Ozaki W, Buchman G. Investigation of the influence of biomechanical force on the ultrastructure of human sagital cranissynostosis. Plast Reconstr Surg 1998;102: 1385-94.

31. Park HD, Min CK, Kwak HH, Youn KH, Choi SH, Kim HJ. Topography of the outer mandibular symphyseal region with reference to the autogenous bone graft. Int J Oral Maxillofac Surg 2004;33:781-785.

32. Sauvigne T, Fusari JP, Monnier A, Breton P, Freidel M. Le prélèvement rètro-Molaire, une alternative au prélèvement mentonnier en chiurgie osseuse préimplantaire: Etude quantitative et qualitative réalisée sur 52 pièces anatomiques. Rev Stomatol Chir Maxillofac 2002; 103:264-8.

33. Hunt DR, Jovanovic SA. Autogenous Bone Harvesting: a Chin Graft Technique for articulate and Monocortical bone Blocks. Int J Periodont Rest Dent 1999;19:165-73.

34. Nkenke E, Schultze-Mosgau S, Radespiel-Tröger M, Kloss FW. Morbidity of harvesting of chin grafts: a prospective study. Clin Oral Impl Res 2001;2:495-502.

35. Schwartz-Arad D, Levin L. Intraoral autogenous Block Onlay Bone Grafting for Extensive Reconstruction of Atrophic Maxillary Alveolar Ridges.J Periodontol 2005; 76:636-41.

36. Roccuzzo M, Ramieri G Spada MC, Bianchi SD, Sid Berrone. Vertical alveolar ridge augmentation by means of a titanium mesh and autogenous bone grafts. Clin Oral Impl Res 2004;5:73-81.

37. Smith MH, Lung KE. Nerve Injuries after dental injection: A Review of the Literature. J Calif Dent Assoc 2006;72:559-64.

38. Ozen T, Orhan D, Gorur I, Ozturk A. Efficacy of Low level laser therapy on neurosensory recovery after injury to the inferior alveolar nerve. Head Face Med. 2006;2:3

39. Robinson PP, Loescher AR, Yates JM, Smith KG. Current management of damage to the inferior alveolar and lingual nerves as a result of removal of third molars. Br J Oral Maxillofac Surg 2004;42:285-92.

40. Euzébio AMZ. Protocolos Clínicos Odontológicos: uso do laser de baixa intensidade. Bons Negócios Ed., $1^{\mathrm{a}}$ ed, 2003, p 5-20.

41. Ladalardo TC, Brugnera Junior A, Bologna ED, Dias PV, Siqueira JTT, Campos RAC. Laserterapia no tratamento de déficit neutossensorial decorrente de procedimento cirúrgico em implantodontia. Implant News $2004 ; 1: 155-8$

42. Kraut RA, Chahal O. Management of patients with trigeminal nerve injuries after mandibular implant placement. JADA 2002;133:1351-4.

43. Misch CM. The Pharmacologic Management of Maxillary Sinus Elevation Surgery. J Oral Implant 1992;15:15-23.

44. Raghoebar MG, Louwerse C, Kalk WWI, Vissink A. Morbidity of Chin Bone Harvesting. Clin Oral Impl Res 2001;2:503-7.

\section{CORRESPONDENCIA}

Prof. Dr. José Ricardo de Albergaria Barbosa

Av. Limeira 901

Caixa Postal 52 - CEP 13414-903

Piracicaba - SP, Brasil.

solate@fop.unicamp.br

barbosa@fop.unicamp.br 